\title{
Simultaneous evaluation of multiple microarray surface chemistries through real-time interferometric imaging
}

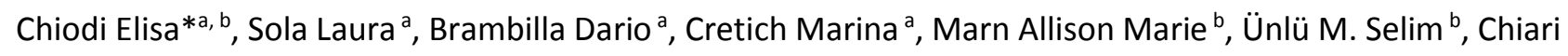
Marcella $^{a}$

\author{
a) Institute of Chemistry of Molecular Recognition \\ National Research Council of Italy \\ Via Mario Bianco 9 \\ 20131 Milan Italy \\ b) Boston University \\ Department of Electrical Engineering \\ Photonics Center \\ 8, St. Mary's Street \\ 02215 Boston (MA) USA
}

* To whom correspondence should be addressed:

Elisa Chiodi

e-mail: elich@bu.edu

telephone: +1 617-206-0221 
Abstract: Surface chemistry is one of the most crucial aspects for microarray modality biosensor development. As a matter of fact, the immobilization capability of the functionalized surface is one of the limiting factors for the final yield of the binding reaction. In this work, we locally deposited many reactive polymers on a single solid support, allowing for a direct comparison of functionality of probes immobilized on different polymers and demonstrating a new way of multiplexing. Our goal was to investigate the immobilization efficiency of reactive polymers, as well as the resulting affinity of the molecular probes, in a single experiment. This idea was demonstrated by spotting a large number of different reactive polymers on an untreated $\mathrm{Si} / \mathrm{SiO}_{2}$ chip, and depositing the same molecular probe on all the spots immediately after. This method proved to be efficient and could be used as an initial qualitative assay to decide which functionalization better suits a certain application. We also showed that the localized functionalization method is applicable to proteins as well as oligonucleotides. Moreover, by means of real-time binding measurements performed with the Interferometric Reflectance Imaging Sensor (IRIS), we demonstrated that this functionalization technique is comparable to the uniformity of classical flat-coating solution. The comparison between the binding curves that were obtained from different polymer spots with the same probe allowed us to decide which polymers would work better to immobilize a model protein, $\alpha$-Lactalbumin, as well as a peptide extracted from the latter, namely LAC-1. The final outcome is promising, and it highlights the multiplexing power of this method: first, it allows to characterize dozens of polymers at once, within a single 60-minutes experiment. Secondly, it removes the limitation, related to coated surfaces, that only molecules with the same functional groups can be tethered to the same solid support. By applying this innovative protocol, there is no more restriction on the type of molecules that can be studied simultaneously and immobilization for each molecular probe can be individually optimized.

Keywords: IRIS; Interferometric Reflectance Imaging Sensor; functional polymers; localized surface functionalization; microarray; binding kinetics; 


\section{Introduction}

Microarrays are analytical devices that work by allowing specific binding between target probes immobilized on the surface (namely, spots) and analyte molecules in solution (MacBeath, 2002). Since microarray-based assays were first introduced in 1995 (Schena et al., 1995), they have rapidly gained popularity in various biological research areas, due to their multiplexing capability and small volume requirement.

The effectiveness of a microarray-based experiment mostly depends on how the probe molecules are immobilized on the surface. As a matter of fact, the chosen immobilization method influences the stability of the molecules as well as their reactivity. For example, if the ligand is not stably bound, it may be lost throughout the experiment. On the contrary, if the probe is immobilized too tightly, it may be less flexible or lose its structure, and therefore have less sites available for binding.

Coating the slide surface with a hydrophilic polymer prior to spotting allows to create a uniform, homogeneous, thin, reactive layer on which the molecules can be immobilized as far from the surface as necessary for them to remain active.

In the last ten years, our group has introduced a family of $\mathrm{N}, \mathrm{N}$-dimethylacrylamide (DMA) based polymers that form a thin film on different materials by a combination of physi- and chemisorption (Pirri et al., 2004). In an effort to tailor surface properties, the precursor of this polymer family, a copolymer of dimethylacrylamide with N-Acryloyloxysuccinimide (NAS) and 3(Trimethoxysilyl)propyl methacrylate (MAPS), has been modified with several building blocks to introduce, for example, azide, alkyne, dibenzocyclooctine, maleimide, functionalities thanks to the reactivity of NAS with amino-groups (Sola et al., 2016). The introduction of other functional groups by post polymerization modification (PPM) reactions and the combination of different monomers during the synthesis has allowed to generate many polymers, ten of which were used in this work (see Figure 1 and Table 1 for monomer mole percent). All these polymers share a common backbone of DMA and MAPS which confers them adhesive properties on a variety of surfaces.

The classical dip and rinse coating approach used with these polymers requires immersing the substrate in their aqueous solution followed by rinse and dry steps (Zilio et al., 2014). Thanks to the adhesive properties of the polymers, this approach, that is considerably simpler than most of the approaches used to produce polymeric coatings, was further simplified by localizing the 
coating through the spotting of only an ultra-small amount of polymer. Theoretically, dozens of different polymers could be arrayed on the surface, thus creating a number of different interfaces between the surface and the probes. Considering how many polymers are available and how differently they react with each probe, deciding which surface chemistry is the optimal one for every experiment is time consuming and laborious, as well as expensive. Indeed, the classical method would include coating many different slides with the different polymers one is willing to test, spotting each of them and performing measurements on them separately. This process results in a copious use of reagents, is time-consuming and may also cause a non-trivial amount of statistical noise due to the experimental conditions that inevitably change for every experiment for example, subtle changes in the temperature or in the $\mathrm{pH}$ of the solutions.

In this work, we combine the new approach for the immobilization of biomolecules based on spotting pL amounts of aqueous solutions of the various copolymers, followed by the deposition, on the same spot, of the biomolecule to be immobilized (Sola et al., 2019) with a real-time binding detection method (Daaboul et al., 2011) recently introduced by our group. This strategy provides an easy, inexpensive and quick assay to test the influence of surface properties on binding affinity in tiny areas on which each probe is anchored on a chemically different environment in a single 60-minute experiment.

\section{Material and methods}

\subsection{Materials}

$\mathrm{N}, \mathrm{N}$-Dimethylacrylamide (DMA), 3-(trimethoxylsilyl)propyl methacrylate (MAPS), $1 \mathrm{H}, 1 \mathrm{H}, 2 \mathrm{H}, 2 \mathrm{H}-$ perfluorodecyl acrylate (PFDA), $\alpha, \alpha^{\prime}$-Azoisobutyronitrile (AIBN), Acrylamido buffer pKa 3.6, acrylamido buffer pKa 8.5, dibenzocyclooctyne-N-hydroxysuccinimidyl ester (DBCO-NHS ester), anhydrous tetrahydrofuran (THF), petroleum ether, dimethylsulfoxide (DMSO), ammonium sulphate $\left(\left(\mathrm{NH}_{4}\right)_{2} \mathrm{SO}_{4}\right)$, phosphate buffered saline (PBS), sucrose monolaurate, Amicon Ultra centrifugal filters (MWCO 3K) and $\alpha$-Lactalbumin were purchased from Sigma Aldrich (St. Louis, MO, USA). Anti $\alpha$-Lactalbumin antibody was purchased from Abcam (Cambridge, UK). All solvents were used as received. $\mathrm{N}$-acryloyloxysuccinimide (NAS) and 3-azido-1-propylamine were synthesized as reported elsewhere (Landi et al., 2010; Mammen et al., 1995). Untreated silicon slides with $110 \mathrm{~nm}$ thermally grown oxide were supplied by Silicon Valley Microelectronics (Santa Clara, CA, USA).

Spotting was performed using a SciFLEXARRAYER S12 (Scienion, Berlin, Germany). 


\subsection{Polymer synthesis}

All polymers, schematically represented in Figure 1, were synthesized as previously reported (Sola et al., 2012, 2016a, 2016b). Briefly, a 20\% w/v total monomer concentration solution was prepared by dissolving each monomer (following the molar ratios reported in Table 1) in anhydrous THF. The solution was degassed for 20 min by purging argon into the monomer solution and then $2 \mathrm{mM}$ of a thermoinitiator (AIBN) was added. The solution was heated for $2 \mathrm{~h}$ at $65^{\circ} \mathrm{C}$ and kept under argon atmosphere. The solution was then diluted 1:1 with anhydrous THF and precipitated in petroleum ether (10 times the volume of the reaction mixture). Polymers were collected as a white powder, and dried under vacuum at room temperature.

Polymers containing charged groups were synthesized using acrylamido derivatives with carboxyl or tertiary amino side chains. These monomers are commercially available under the trade name of Acrylamido Buffer. The pKa of their ionizable groups covers the entire $\mathrm{pH}$ scale. The Acrylamido buffer with pKa 3.6 was used for the negative polymer, while the one with pKa 8.5 was chosen for the positive polymer. To synthesize the amphoteric polymer, a mixture of these acrylamido buffers was used.

Azide functionalities were introduced in parent polymers containing $\mathrm{N}$-Acryloyloxysuccinimide (NAS) via post-polymerization modification, as described elsewhere (Sola et al., 2016). Essentially the copolymer was dissolved in anhydrous THF to a final concentration of $20 \% \mathrm{w} / \mathrm{v}$; a 2.5 molar excess, respect to the molar ratio of NAS, of 3-azido-1-propylamine was added to the crude material. The mixture was stirred for $5 \mathrm{~h}$ at room temperature under argon atmosphere and then diluted 1:1 with anhydrous THF. The polymers were precipitated in petroleum ether (10 times the volume of the reaction mixture), filtered on a Buchner funnel and dried under vacuum at room temperature. 


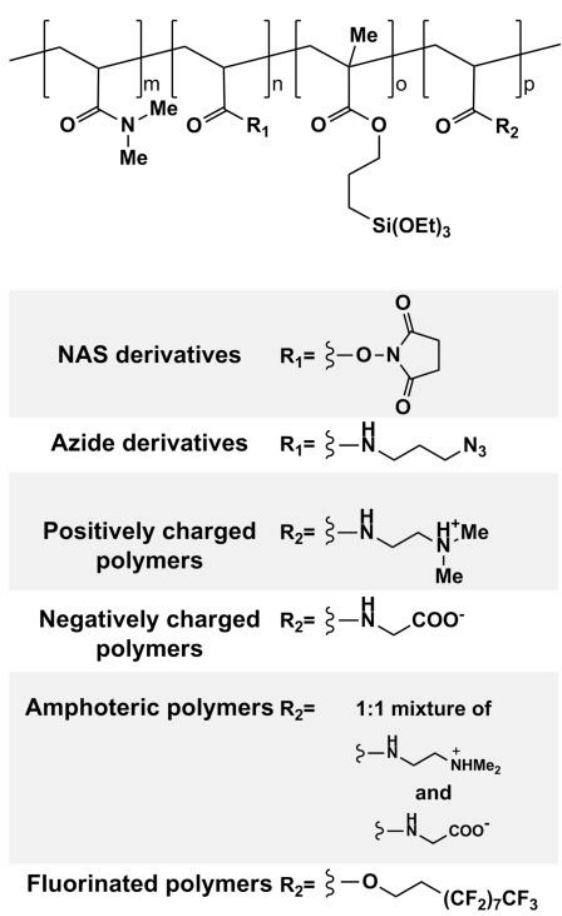

Figure 1: Chemical structure of tested copolymers, see Table 1 for monomer mole percentages.

Table 1: Mole percent of monomers used to synthesize polymers.

\begin{tabular}{|lcccc|}
\hline Copolymer & \multicolumn{4}{c|}{ Monomer mole percentage } \\
\cline { 2 - 5 } & $\mathbf{m}$ & $\mathbf{n}$ & $\mathbf{0}$ & $\mathbf{p}$ \\
\hline Copoly NAS 10\% & 89 & 10 & 1 & - \\
Copoly NAS 10\% positive & 87 & 10 & 1 & 2 \\
Copoly NAS 10\% negative & 87 & 10 & 1 & 2 \\
Copoly NAS 10\% amphoteric & 87.5 & 10 & 0.5 & 2 \\
Copoly Fluoride & 94 & 2 & 1 & 3 \\
Copoly Azide & 97 & 2 & 1 & - \\
Copoly Azide 10\% & 89 & 10 & 1 & - \\
Copoly Azide 10\% negative & 87 & 10 & 1 & 2 \\
Copoly Azide 10\% amphoteric & 87.5 & 10 & 0.5 & 2 \\
Copoly Azide Fluoride & 94 & 2 & 1 & 3 \\
\hline
\end{tabular}

\subsection{Synthesis of DBCO-modified $\alpha$-Lactalbumin}

$3.53 \mu \mathrm{L}$ of $40 \mathrm{mM}$ solution of DBCO-NHS ester in DMSO (10 equivalents) were added to $100 \mu \mathrm{L}$ of 1 $\mathrm{mg} / \mathrm{mL} \alpha$-Lactalbumin in PBS and allowed to react for $30 \mathrm{~min}$ at room temperature. At the end, 10 $\mu \mathrm{L}$ of TRIS-HCl $1 \mathrm{M} \mathrm{pH} 8$ were added to quench the reaction. After 5 min of quenching, the reaction mixture was filtered on Amicon Ultra $3 \mathrm{~K}$ centrifugal filter for $2 \mathrm{~min}$ at 12,000xg to remove unreacted DBCO-NHS ester. PBS was finally added to the DBCO-modified $\alpha$-Lactalbumin solution 
to a final volume of $100 \mu \mathrm{L}$, and the solution was deposited on silicon slides without further purification.

\subsection{Synthesis of LAC-1 peptides}

LAC-1 peptides synthesis is described elsewhere (Gori et al., 2017). Sequences are reported in Table S1. Briefly, the peptides were assembled on a 2-CTC resin by a microwave-assisted Fmoc solid-phase peptide synthesis (SPPS) protocol, on a Biotage ALSTRA Initiator + Peptide synthesizer. Chain elongation was performed by iterative amino acids coupling cycles and Fmoc deprotection. Peptides were then cleaved from the resin and purified by RP-HPLC.

\subsection{IRIS system}

The Interferometric Reflectance Imaging Sensor (IRIS) uses optical phase difference to detect very small amounts of biomass that accumulate on an active surface (Figure S1). The measurement concept and the setup were fully described in Daaboul et al. (2010) and Needham et al. (2019). Briefly, the active surface is composed of a silicon chip with a thermally grown $\mathrm{SiO}_{2}$ layer on top. The chip contains two holes through the Si substrate to allow the passage of liquid across the active surface to conduct dynamic binding measurements. The layered sensor structure creates a common path interferometer where the reflectance at a particular wavelength depends directly on the thickness of the top transparent layer. In our system, the sample can be illuminated by four independent LEDs with a peak at four different visible wavelengths ( $455 \mathrm{~nm}, 518 \mathrm{~nm}, 598 \mathrm{~nm}$ and $635 \mathrm{~nm}$ ). By measuring the reflectance from the substrate at distinct wavelengths across the visible spectrum, we are able to reconstruct the reflectance curve from the layered substrate and deduce the thickness of the transparent layer with pm accuracy. Changes in the optical path length due to the capture of a target of interest on the $\mathrm{SiO}_{2}$ layer causes a measurable shift in this reflectance curve, and we are able to achieve $\mathrm{pg} / \mathrm{mm}^{2}$ sensitivity to target binding (Daaboul et al., 2010). However, continuously acquiring images with each of these four illumination wavelengths throughout the experiment, and then calculating the reflectance curve for each four-color acquisition has practical limitations. Therefore, we start from the assumption that during the whole experiment the thickness varies in such a small range $( \pm 10 \mathrm{~nm})$ that it can be directly correlated to the change in reflectivity (Sevenler and Ünlü, 2016). This way, we can acquire one single image in each of the four available LEDs at the beginning of the experiment, calculate the reflectance curve, and then use the resulting reflectance curve to create a look-up table for the 
conversion from reflectance to biomass thickness. The images were acquired with a simple CCD camera (PointGrey) and analyzed through a custom-made ImageJ plugin and a MATLAB software. More details about the conversion from reflectance values to mass density can be found elsewhere (Daaboul et al., 2010, Sevenler and Ünlü, 2016).

\subsection{Chip surface functionalization}

The experiments were performed on $25 \times 12.5 \mathrm{~mm}$ silicon chips (Figure S2a), purchased from Silicon Valley Microelectronics (Santa Clara, CA) with a 110nm layer of silicon oxide grown on top. Chips were spotted with a SciFlexArrayer S12 spotter (Scenion, Berlin, Germany). For what concerns localized functionalization samples, polymer solutions were prepared by diluting them up to $0.014 \%$ in water with sucrose monolaurate $0.01 \% \mathrm{w} / \mathrm{v} .400 \mathrm{pL}$ of these solutions were spotted on untreated IRIS chips. Then, for the protein microarrays, $400 \mathrm{pL}$ of either native or DBCO modified $\alpha$-Lactalbumin $1 \mathrm{mg} / \mathrm{mL}$ were spotted on top of that. Instead, for the peptide microarray, LAC-1 peptides were first dissolved in DMSO at a stock concentration of 1mM. LAC-1 was then diluted in PBS at a concentration of 100uM, while LAC-1-Prg was diluted at the same concentration into the printing buffer suitable for CuACC conjugation: $25 \mathrm{mM} \mathrm{Na}$ /Acetate p4.8, $15 \mathrm{mM}$ trehalose, 100 mM CuSO4, 400 mM THPTA and 6.25 mM Ascorbic Acid. 400pL of peptide solutions obtained this way were finally deposited on top of polymer-spotted chips.

In both cases, the spotted chips were left overnight in a $\mathrm{NaCl}$ saturated humid chamber. The chips were washed with a $0.5 \% \mathrm{w} / \mathrm{v}$ water solution of a copolymer of dimethylacylamide and allyl glycidyl ether (EPDMA) synthesized as reported elsewhere (Chiari et al., 2000). This polymer, originally developed to suppress electroosmotic flow in capillary electrophoresis is known for its ability to form a thin layer on glassy surface with antifouling properties by dip and rinse approach. Some experiments for this work were also performed in a flat coating configuration. For these experiments, the samples were prepared in a different way. Briefly, the surface of the chips was first oxygen plasma activated for 10 minutes, and then the chips were immersed in the aqueous polymer solutions ( $1 \%, \mathrm{w} / \mathrm{v}$ polymer in an aqueous solution of $20 \%$ saturated ammonium sulfate) for 30 minutes. Afterwards, the substrates were washed with DI water and dried under nitrogen stream. Finally, to complete the coating procedure, they were baked in a stove at $80^{\circ} \mathrm{C}$ for 15 minutes. The chips were then spotted with $400 \mathrm{pL}$ of native $\alpha$-Lactalbumin $1 \mathrm{mg} / \mathrm{mL}$. As above, the spotted chips were left overnight in a $\mathrm{NaCl}$ saturated humid chamber. In this case, a one-hour 
blocking step with a 50mM ethanolamine solution was performed to inactivate the residual amine groups on the surface.

\subsection{Microfluidic chamber}

Prior to starting the experiment, every chip (Figure S2a, S2b) was topped with an adhesive spacer and an AR coated glass slide to form a chamber for in-liquid measurements. The glass was purchased from Abrisa Technology (Torrance, CA) while the spacer was customized for us by Grace BioLabs (Bend, OR). The volume of the chamber obtained this way was less than $5 \mathrm{uL}$. The whole system has a dead volume of less than 500uL.

\section{Results and discussion}

\subsection{Localized surface functionalization: testing many surface chemistries on the same chip}

The adhesive characteristics of DMA, MAPS copolymers allow to generate films on localized regions of the substrate by spotting $\mathrm{pL}$ amounts of polymer solutions. Two sets of polymers were used in this experiment: one contains succinimide esters while the second one contains azide groups. In each series, three variants were generated by copolymerizing, in addition to the backbone monomers, acrylic monomers bearing carboxyl or tertiary amine groups or a mixture of both monomers in such a ratio to define a precise isoelectric point. At the $\mathrm{pH}$ used for the hybridization, the surface is negatively or positively charged in the first two cases. The net charge of the amphoteric surface can be tuned from negative to positive depending on the $\mathrm{pH}$ of the hybridization buffer. In the experimental conditions used here it has an overall negative charge. In addition, the charge can be modulated by tailoring the ratio of the two acrylamido buffers in the polymer or by selecting different acrylamido buffers pairs.

The parent polymers, Copoly NAS $10 \%$ and its derivative Copoly Azide are neutral or only negligibly charged; they bind either native proteins by nucleophile reaction between succinimidyl esters and amino groups naturally present in proteins, or DBCO modified proteins by azide-alkyne strain promoted cicloaddition, a classical click chemistry reaction.

As mentioned above, the immobilization technique detailed here offers a set of advantages. First of all, it allows the user to save time by avoiding the whole time-taking process of coating the slide. Moreover, a much smaller quantity of polymer is needed with respect to flat-coating. Finally, a single experiment can provide information on the performance of multiple surface chemistries at a time. 
In Figure $2 \mathrm{a}$ a schematic representation of the functionalization is reported, compared to the classic flat coating solution (Figure $2 \mathrm{~b}$ ). In a single spotting section, aqueous solutions of ten polymers at $0.014 \% \mathrm{w} / \mathrm{v}$ were spotted in array format. On top of each polymer the same protein, $\alpha$-Lactalbumin (either native or DBCO-modified) at a concentration of $1 \mathrm{mg} / \mathrm{mL}$ was deposited. To avoid non specific adsorption of biomolecules on the area surrounding the spots, after deposition of the capture probe, a blocking step was performed using a hydrophilic polymer, EPDMA, previously reported by our group (Chiari et. al, 2000). An IRIS image of the dry chip is displayed in Figure 2c. Distinct groups of spots show different levels of brightness, and therefore highlight a variability in mass density. The brighter spots are indeed a result of the better immobilization capacity of the polymer underneath.

(a)

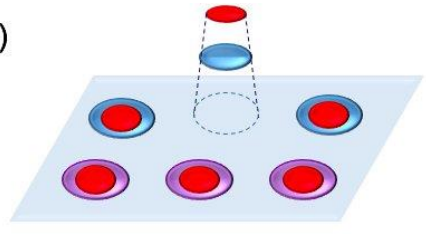

(b)

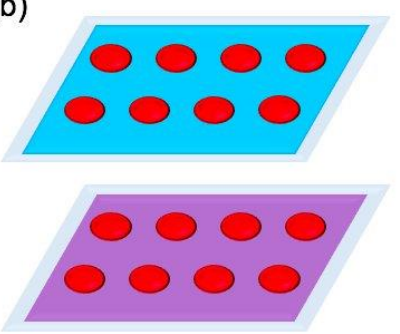

(c)

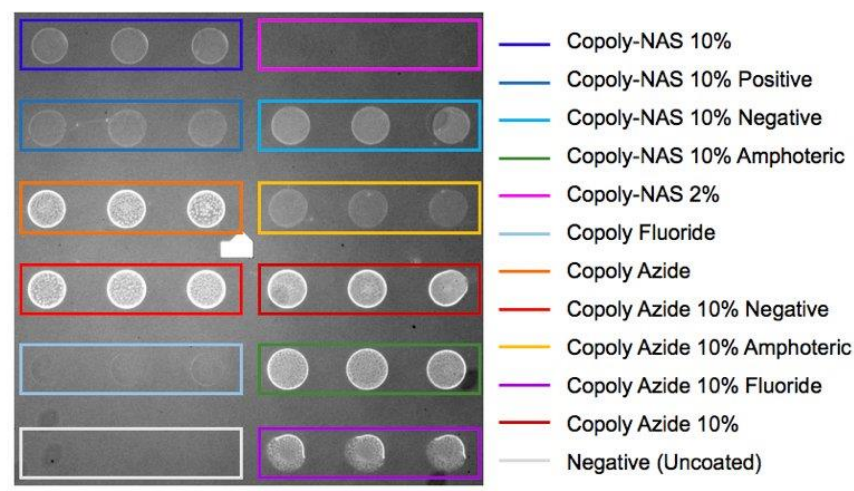

Figure 2 - On the left, a scheme of the spotted polymers technique (a) and flat coating technique (b). On the right, a dry IRIS chip image acquired before starting the real-time binding experiment. On the chip a $6 \times 6$ matrix of $\alpha$ Lactalbumin spots is visible. Each group of three spots corresponds to a different polymer (c).

The chip was used to run a 60-minutes long in-liquid IRIS experiment. First, filtered PBS buffer was flowed into the chamber for 15 minutes, allowing the system to stabilize. Afterwards, anti- $\alpha$ Lactalbumin antibody at $1 \mathrm{ug} / \mathrm{mL}(\approx 7 \mathrm{nM})$ was injected for 20 minutes at an average speed of 200uL/min. PBS buffer was then injected as a washing buffer for another 20 minutes. Finally, the surface was restored by flowing a $100 \mathrm{mM}$ glycine solution at $\mathrm{pH}=2$ for 5 minutes. During this whole experiment, interferometric images were acquired. Each point of the curve in Figure 3 corresponds to one acquired image. To get a better signal-to-noise ratio, each image is the average of 100 acquired frames. 


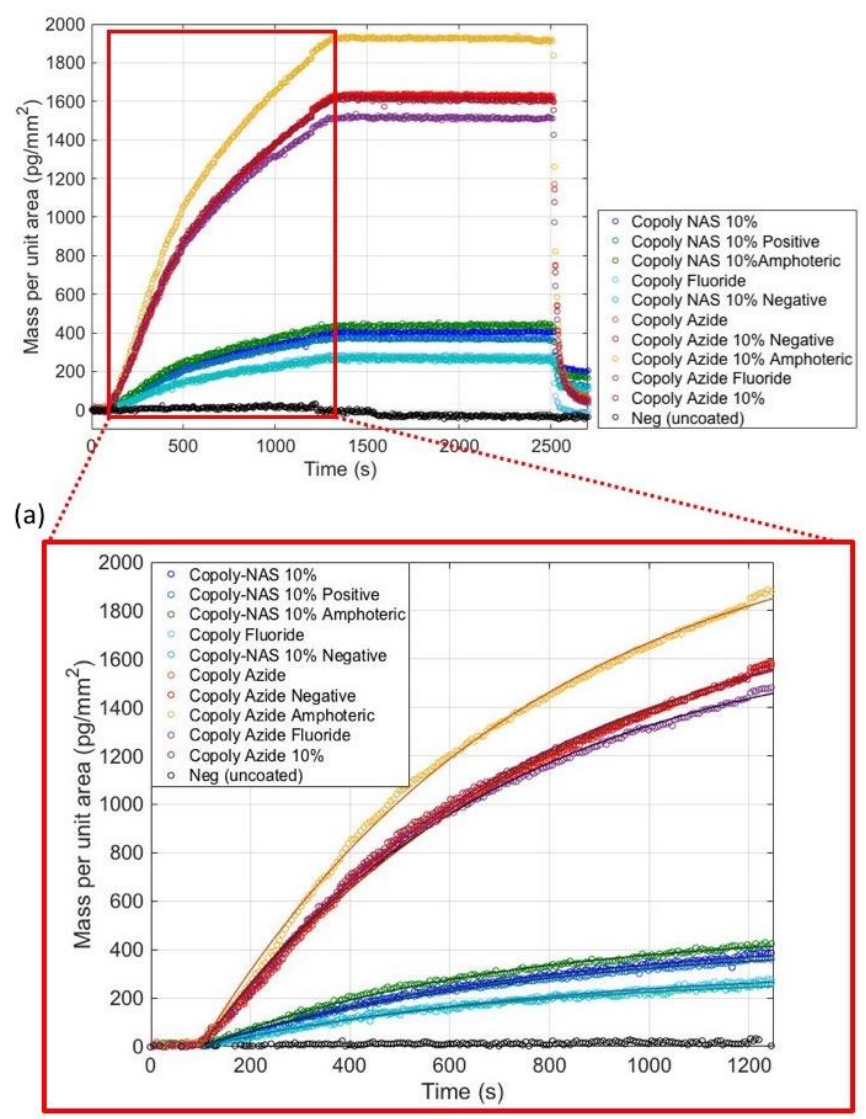

Figure 3 - Anti- $\alpha$-Lactalbumin binding to two different modifications of $\alpha$-Lactalbumin spotted on a single IRIS chip. The solid lines in the inset (a) show a classic Langmuir fit of the data.

By analyzing the first 20 minutes of data, as detailed in par. 2.2, the binding curves in figure 3a were obtained. A simple Langmuir one-to-one binding equation was used to fit the data (eq.1).

$$
d m(t)=m_{e q} \cdot \frac{k_{O N} C}{k_{O N} C+k_{O F F}} \cdot\left(1-e^{-\left(k_{O N} C+k_{O F F}\right) \cdot t}\right)
$$

where $d m(t)$ indicates the differential mass density, $t$ is the time in seconds, $m_{e q}$ is the mass density value reached at the equilibrium, $k O N$ is the association rate, KOFF is the dissociation rate and $C$ is the concentration of the analyte in solution, that, in our case, is around $7 \mathrm{nM}$.

This way, we could estimate the dynamic constants of the reactions. The parameters obtained from this analysis are reported in Table 2. 
Table 2: ON and OFF rates obtained through a classic Langmuir fit of anti- $\alpha$-Lactalbumin binding on different $\alpha$-Lactalbumin modifications, spotted on top of different polymers spots.

\begin{tabular}{|lccc|}
\hline Copolymer & KoN $\left(\mathbf{1 0}^{\mathbf{4}} \mathbf{M}^{-1} \mathbf{s}^{-1}\right)$ & Koff $_{\left(10^{-4} \mathbf{s}^{-1}\right)}$ & $\mathrm{K}_{\mathrm{D}}\left(\mathbf{1 0 ^ { - 8 }} \mathbf{M}\right)$ \\
\hline Copoly NAS 10\% & $9.4 \pm 0.2$ & $7.2 \pm 0.1$ & $0.77 \pm 0.02$ \\
Copoly NAS 10\% positive & $9.0 \pm 0.1$ & $7.7 \pm 0.3$ & $0.86 \pm 0.04$ \\
Copoly NAS 10\% negative & $8.3 \pm 0.2$ & $9.7 \pm 0.6$ & $1.17 \pm 0.08$ \\
Copoly NAS 10\% amphoteric & $11.1 \pm 0.2$ & $6.5 \pm 0.1$ & $0.59 \pm 0.01$ \\
Copoly Fluoride & $6.5 \pm 0.1$ & $8.8 \pm 0.4$ & $1.35 \pm 0.07$ \\
Copoly Azide & $7.7 \pm 0.1$ & $8.4 \pm 0.3$ & $1.09 \pm 0.04$ \\
Copoly Azide 10\% negative & $7.5 \pm 0.7$ & $7.8 \pm 0.2$ & $1.04 \pm 0.10$ \\
Copoly Azide 10\% amphoteric & $9.6 \pm 0.9$ & $8.2 \pm 0.3$ & $0.85 \pm 0.09$ \\
Copoly Azide 10\% Fluoride & $8.0 \pm 0.8$ & $9.0 \pm 0.3$ & $1.13 \pm 0.12$ \\
Copoly Azide 10\% & $9.8 \pm 0.1$ & $8.8 \pm 0.2$ & $1.00 \pm 0.03$ \\
\hline
\end{tabular}

As it can be noticed from Figure 3, the two families of polymers are clearly distinct in terms of dynamic behavior and equilibrium points reached.

However, this preliminary result mainly depends on the mass density value at the beginning of the experiment - that is, the immobilized biomaterial that remains on the surface after spotting. Indeed, a significant correlation exists between the equilibrium point reached by each curve and the average initial mass per spot (Table 3).

Table 3: Initial mass density values for the $\alpha$-Lactalbumin spots on different polymers.

\begin{tabular}{|lc|}
\hline Copolymer & Initial mass density $\left(\mathbf{n g} / \mathbf{m m}^{2}\right)$ \\
\hline Copoly NAS 10\% & $1.36 \pm 0.02$ \\
Copoly NAS 10\% positive & $0.66 \pm 0.02$ \\
Copoly NAS 10\%negative & $1.17 \pm 0.03$ \\
Copoly NAS 10\% amphoteric & $1.09 \pm 0.06$ \\
Copoly Fluoride & $0.73 \pm 0.04$ \\
Copoly Azide & $1.69 \pm 0.07$ \\
Copoly Azide 10\% negative & $3.92 \pm 0.03$ \\
Copoly Azide 10\% amphoteric & $3.83 \pm 0.04$ \\
Copoly Azide 10\% Fluoride & $2.89 \pm 0.04$ \\
Copoly Azide 10\% & $1.93 \pm 0.03$ \\
Negative (Uncoated) & $-0.69 \pm 0.05$ \\
\hline
\end{tabular}


To quantitatively understand this correlation, we calculated the correlation coefficient between the initial mass on the surface and the equilibrium points reached, as in equation 2:

$\rho_{m_{0}, m_{e q}}=\frac{\operatorname{cov}\left(m_{0}, m_{e q}\right)}{\sigma_{m_{0}} \cdot \sigma_{m_{e q}}}$

This calculation led to a correlation coefficient value of 0.85 , which can be considered significant (Figure S3).

Measuring how much mass the polymers immobilized is both interesting and informative, and we did so with DNA in our previous work on this topic (Sola et. al., 2019). However, another really important data we are interested in is how reactive the immobilized molecules are. A good approach would be to normalize the curves above by dividing them for the initial mass value (Figure 4).

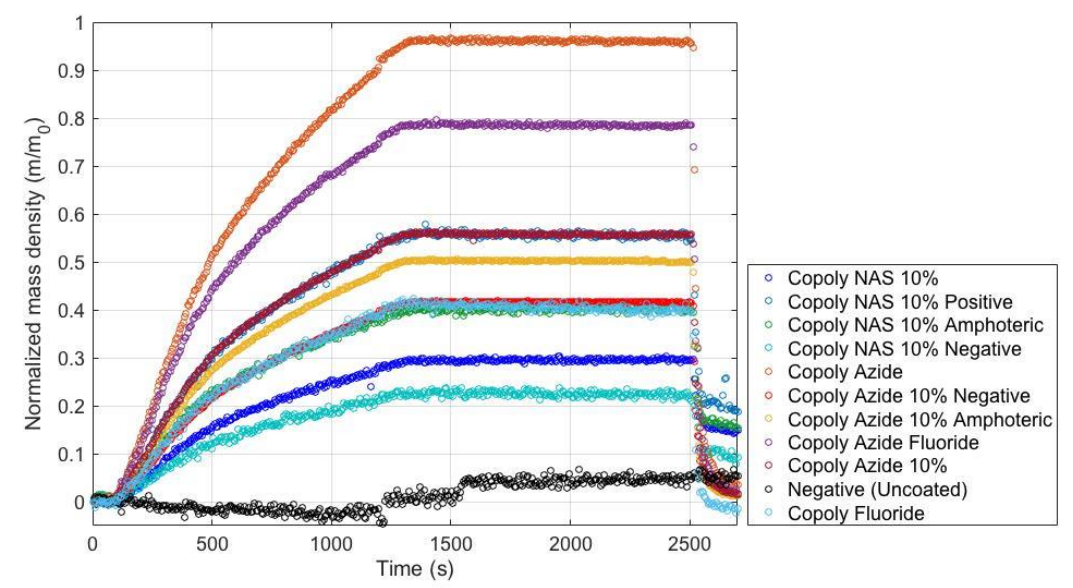

Figure 4: The curves in figure 3a, normalized by dividing every curve for the initial mass value.

This normalization shows that having a large quantity of biomatierial immobilized on the surface is not always a good indication of the high reactivity of the probes. Indeed, even if the overall binding is higher on the Copoly Azide 10\% amphoteric spot (Figure 3a), in the end the neutral Copoly Azide spot almost doubles its mass density $\left(\mathrm{m} / \mathrm{m}_{0} \approx 1\right.$, Figure 4$)$, while the former accumulates less than half of the initial mass value $\left(\mathrm{m} / \mathrm{m}_{0} \approx 0.5\right)$. A possible explanation for this is that the presence of charges could inhibit the binding of this specific antibody due to electrostatic repulsion. Another possibility is that the charged polymers immobilize the probe so tightly that they modify its structure and therefore affect its reactivity. This second possibility is less probable, since $\alpha$-Lactalbumin is quite symmetric and the charges are well distributed on its surface. 
To summarize, even though the neutral polymers retain less mass from spotting (Table 2, Figure 3), the molecules that are immobilized onto those show a higher reactivity (Figure 4).

\subsection{Comparison to flat coating}

To further demonstrate that our assay is comparable to the classical flat-coating method, in terms of qualitative performance of the polymers, we chose two of the polymers mentioned above, namely Copoly NAS 10\% amphoteric and Copoly Fluoride, and we performed the same measurements in a flat coating configuration.

First, two chips were coated with, respectively, Copoly Fluoride and Copoly NAS 10\% amphoteric, with the classical flat coating procedure as described in the Material and Methods section (Par. 2.3).

On both chips, the same IRIS experiment described above was performed: the chamber was mounted, with the glass and spacer, and the obtained cartridge was positioned inside the instrument. PBS buffer was flowed into the chamber for 15 minutes to stabilize the system. Afterwards, anti $\alpha$-Lactalbumin $1 \mathrm{ug} / \mathrm{mL}(\approx 7 \mathrm{nM})$ was injected and recirculated for 20 minutes at an average speed of $200 \mathrm{uL} / \mathrm{min}$. A 20 -minute washing with PBS was performed, and a 5-minute surface restoration with a $100 \mathrm{mM}$ glycine solution $(\mathrm{pH}=2)$ was used to go back to the starting point. 


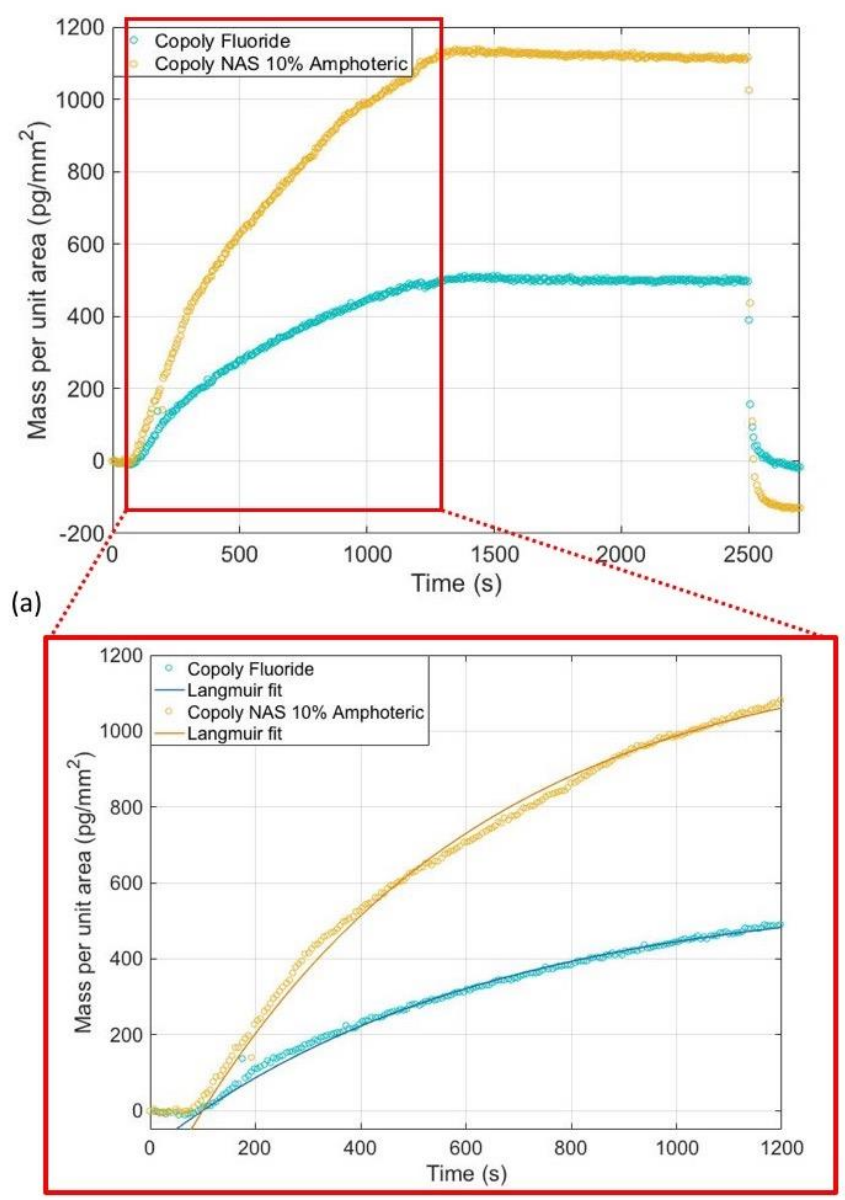

Figure 5: Anti- $\alpha$-Lactalbumin binding to $\alpha$-Lactalbumin spotted on two flat-coated IRIS chips: one coated with Copoly Fluoride (light blue curve) and one with Copoly NAS 10\% Amphoteric (yellow). The solid lines in the inset (a) show a classic Langmuir fit of the data.

As for the experiment described in Paragraph 3.1, every point of the curves displayed in Figure 5 corresponds to the average of 100 acquired frames.

The parameters (Table S2) and the curves (Figure 5) obtained show that these two polymers qualitatively behave the same in both configurations. Indeed, by comparing Figure 3 and Figure 5, one can notice that in both cases the equilibrium point reached by the Copoly Fluoride curve is sensibly lower than the one reached by Copoly NAS 10\% amphoteric.

Moreover, the initial mass values can be compared in the two cases (Table S3), and it can be observed that they are in good agreement.

To investigate the problem in depth, we normalized these data the same way we did above, by dividing the curves for the initial mass value (Figure S4). The result confirmed the previous findings: the two polymers behave the same way in terms of reactivity, as it also emerges from the data in Figure 4, where the Copoly Fluoride curve is following the same path as the Copoly NAS $10 \%$ amphoteric one. 


\subsection{Localized immobilization of LAC-1 peptide}

In order to further demonstrate the power of the localized functionalization method, we applied it to the immobilization and functional test of a model peptide, LAC-1, which is an epitope belonging to the $\mathrm{N}$-terminal part of $\alpha$-Lactalbumin protein. This epitope is the most reactive of the characterized series (Hochwallner et al., 2010) and was synthesized in two modifications, as described in Material and Methods (Par. 2.4).

We have previously shown that the chemistry used to immobilize peptides has a crucial role on their reactivity (Gori et al., 2016) by demonstrating that immobilization of a collection of peptides on NAS copolymers resulted in a loss of reactivity of the probes when the microarray was incubated with human serum from cystic fibrosis (CF) patients affected by Burkholderia infections. On the other hand, when the same peptides were immobilized on Copoly Azide through a clickchemistry reaction, their binding affinity towards serum antibodies was enhanced providing discrimination between healthy and affected individuals in a serological assay for Burkholderia. Therefore, peptides are good candidates for our method to show the difference that surface chemistry can make on the reactivity of biomolecules. Focusing on the model peptide chosen here, we (Gori et al., 2017) also showed that the reactivity of LAC-1 in fluorescence measurements is crucially related to the spacing between the probes, its availability to bind being diminished by overcrowding effects.

We spotted an IRIS chip according to the protocol described in Material and Methods (Par. 2.6). In Figure S5 an image of the dry IRIS chip can be observed. One can notice that LAC-1 spots on different polymers, diversely from $\alpha$-Lactalbumin ones (Figure 2c), look almost uniformly bright. This is an indication of the fact that the initial mass on all the spots is similar (Table S4). We performed IRIS real-time measurements on this chip to detect the binding of anti- $\alpha$ Lactalbumin antibody. The experiment was carried out in an identical manner as the one described in Par. 3.1., except for antibody concentration, which was higher: $5 \mathrm{ug} / \mathrm{mL}$ ( $\approx 35 \mathrm{nM})$. The peptide has indeed a lower binding affinity to the antibody with respect to the protein (Hochwallner et al., 2010). 
(a)

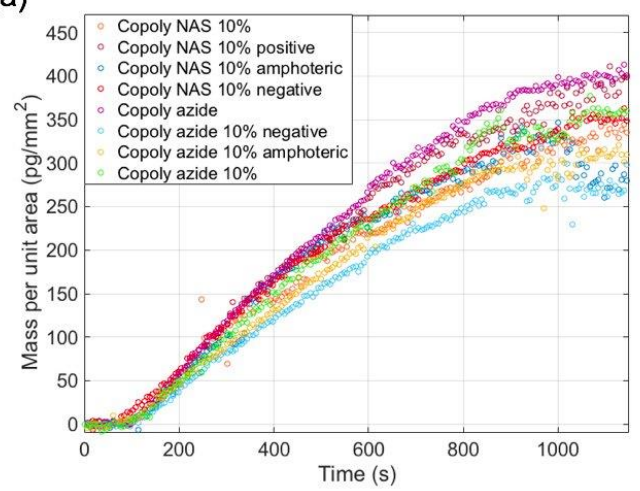

(b)

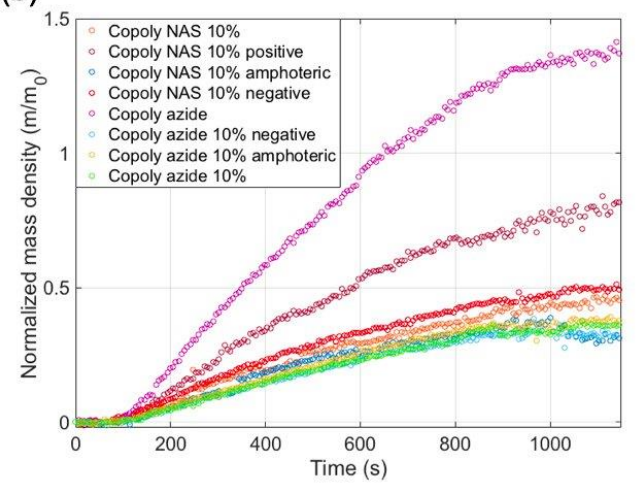

Figure 6 - Application of the localized functionalization method to the immobilization of LAC-1 peptide. (a) Anti- $\alpha$ Lactalbumin binding to two different modifications of LAC-1 spotted on a single IRIS chip. Each curve corresponds to a different spotted polymer. (b) The curves in Figure 6a, normalized by dividing each of them by the initial mass density value.

By analyzing the images acquired through the IRIS setup, as already described in Par. 3.1, we obtained the binding curves displayed in Figure 6a. Noticeably, not much difference is observable between the curves. However, when normalizing for the initial mass value (Figure 6b), Copoly Azide curve stands out. This corroborates our previous findings that Copoly Azide is the most suitable polymer for maintaining a high reactivity of the peptide, while immobilizing an adequate quantity (Gori et al., 2016). Moreover, having a higher concentration of the Azide copolymer on the surface does not seem to increase binding affinity; instead, it decreases it. This could be due to overcrowding effects, as we previously hypothesized (Gori et al., 2017), where, we related the spacing between probes on the surface with their reactivity.

The calculation of the correlation between the initial mass density value and the equilibrium point reached by the curves (eq.2) yielded a negative value for the correlation coefficient $(\rho=-0.59)$, indicating that, in this specific case, the immobilization capacity of the polymers is uncorrelated with the reactivity of the probes (Figure S6).

Interestingly, the charged Azide copolymers behave similarly to the NAS ones; this could be related to the negative overall charge that they assume in these conditions, which could inhibit binding due to electrostatic repulsion. This hypothesis is also validated by the fact that the positively charged $10 \%$ NAS copolymer has an increased reactivity with respect to the other members of the same family.

\section{$4 \quad$ Conclusions}


In summary, we pursued the effort started in our latest work (Sola et. al., 2019) of developing an innovative assay that allows to simultaneously test the performance of different surface chemistries, localized on distinct regions of the same support. Most importantly, we demonstrated that this immobilization technology can successfully be applied to proteins, as well as oligonucleotides, which is not trivial and could be challenging due to the complexity of protein structure. Briefly, we precisely quantified both the initial mass immobilized on each localized polymer spot as well as the final yield of the reaction. Moreover, we calculated the correlation between those values and, thanks to the real-time binding measurements, we could estimate the actual reactivity of the probes by applying a simple normalization. Single 60-minute experiments were therefore enough to collect all the information that is necessary to qualitatively compare the performance of the different polymers. The results were in optimal agreement with those obtained in the classical flat-coating configuration. We therefore showed that the combination of this localized functionalization technology and the real-time, in liquid IRIS technique allows the user to quickly understand which surface chemistry would better suit any specific application. Furthermore, it removes the limitation regarding the number of molecules with different chemistries that can be immobilized on the same support.

\section{$5 \quad$ Acknowledgements}

This work was partially funded by European Union's Horizon 2020 research and innovation program under grant agreement $\mathrm{n}^{\circ} 766466$ (INDEX); and by Regione Lombardia, project AMANDA (Abnormal Metabolic states, cellular stressors and NeuroDegenerative processes). 
Chiari., M., Cretich., M., Damin., F., Ceriotti., L., Consonni., R., 2000. New adsorbed coatings for capillary electrophoresis, Electrophoresis, 21, 909-916.

https://doi.org/10.1002/(SICI)1522-2683(20000301)21:5<909::AID-ELPS909>3.0.CO;2-L

Daaboul, G.G., Vedula, R.S., Ahn, S., Lopez, C.A., Reddington, A., Ozkumur, E., Ünlü, M.S., 2011. LED-based Interferometric Reflectance Imaging Sensor for quantitative dynamic monitoring of biomolecular interactions. Biosens. Bioelectron. 26, 2221-2227.

https://doi.org/10.1016/j.bios.2010.09.038

Gori, A., Sola, L., Gagni, P., Bruni, G., Liprino, M., Peri, C., Colombo, G., Cretich, M., Chiari, M., 2016. Screening Complex Biological Samples with Peptide Microarrays: The Favorable Impact of Probe Orientation via Chemoselective Immobilization Strategies on Clickable Polymeric Coatings. Bioconjug. Chem. 27, 2669-2677.

https://doi.org/10.1021/acs.bioconjchem.6b00426

Gori, A., Cretich, M., Vanna, R., Sola, L., Gagni, P., Bruni, G., Liprino, M., Gramatica, F., Burastero, S., Chiari, M., 2017. Multiple epitope presentation and surface density control enabled by chemoselective immobilization lead to enhanced performance in IgE-binding fingerprinting on peptide microarrays. Anal. Chim. Acta 983, 189-197.

https://doi.org/10.1016/i.aca.2017.06.027

Hochwallner, H., Schulmeister, U., Swoboda, I., Focke-Tejkl, M., Civaj, V., Balic, N., Nystrand, M., Härlin, A., Thalhamer, J., Scheiblhofer, S., Keller, W., Pavkov, T., Zafred, D., Niggemann, B., Quirce, S., Mari, A., Pauli, G., Ebner, C., Papadopoulos, N.G., Herz, U., van Tol, E.A.F., Valenta, R., Spitzauer, S., 2010. Visualization of clustered IgE epitopes on $\alpha$-lactalbumin. J. Allergy Clin. Immunol. 125. https://doi.org/10.1016/i.jaci.2010.03.007

Landi, F., Johansson, C.M., Campopiano, D.J., Hulme, A.N. , 2010. Synthesis and application of a new cleavable linker for "click"-based affinity chromatography. Org. Biomol. Chem., 8, 56-59. https://doi.org/10.1039/b916693a

MacBeath, G., 2002. Protein microarrays and proteomics. Nature Genetics, 32 (Suppl.), 526-532. https://doi.org/10.1038/ng1037

Mammen, M., Dahmann, G., Whitesides, G.M., 1995. Effective inhibitors of hemagglutination by influenza virus synthesized from polymers having active ester groups. Insight into mechanism of inhibition. J. Med. Chem., 38, 4179-4190.

https://doi.org/10.1021/im00021a007

Needham, J., Lortlar Ünlü, N., Ünlü, M. S., 2019. Interferometric Reflectance Imaging Sensor (IRIS) for Molecular Kinetics with a Low-Cost, Disposable Fluidic Cartridge, in: Fitzgerald J., Fenniri H. (eds) Biomimetic Sensing. Methods in Molecular Biology, vol 2027. Humana, New York, NY, pp. 1528.

Pirri, G., Damin, F., Chiari, M., Bontempi, E., and Depero, L. E., 2004. Characterization of A Polymeric Adsorbed Coating for DNA Microarray Glass Slides. Anal. Chem. 76, 1352-1358. https://doi.org/10.1021/ac0352629 
Schena, M., Shalon, D., Davis, R. W., Brown, P. O., 1995. Quantitative monitoring of gene expression patterns with a complementary DNA microarray. Science, 270, 467-470 https://doi.org/10.1126/science.270.5235.467

Sevenler D., Ünlü M.S., 2016. Numerical techniques for high-throughput reflectance interference biosensing. Journal of Modern Optics, 63, 1115-1120.

http://doi.org/10.1080/09500340.2015.1117668

Sola, L., Chiari, M., 2012. Modulation of electroosmotic flow in capillary electrophoresis using functional polymer coatings. J. Chromatogr. A., 1270, 324-329.

https://doi.org/10.1016/i.chroma.2012.10.039

Sola, L., Damin, F., Cretich, M., Chiari, M., 2016. Novel polymeric coatings with tailored hydrophobicity to control spot size and morphology in DNA microarray. Sensor. Actuator. B Chem., 231, 412-422.

https://doi.org/10.1016/j.snb.2016.03.049

Sola, L., Damin, F., Gagni, P., Consonni, R., Chiari, M, 2016. Synthesis of clickable coating polymers by post-polymerization modification: application in microarray technology. Langmuir, 32, 1028410295.

https://doi.org/10.1021/acs.langmuir.6b02816

Sola, L., Damin, F., Chiari, M., 2019. Array of multifunctional polymers for localized immobilization of biomolecules on microarray substrates. Anal. Chim. Acta 1047, 188-196.

https://doi.org/10.1016/i.aca.2018.10.006

Zilio, C., Sola, L., Damin, F., Faggioni, L., and Chiari, M., 2014. Universal hydrophilic coating of thermoplastic polymers currently used in microfluidics. Biomed. Microdevices 16, 107-114.

https://doi.org/10.1007/s10544-013-9810-8 


\section{List of figures}

Figure 1: Chemical structure of tested copolymers, see Table 1 for monomer mole percentages.

Figure 2: On the left, a scheme of the spotted polymers technique (a) and flat coating technique (b). On the right, a dry IRIS chip image of the $\alpha$-Lactalbumin spotted chip acquired before starting the real-time binding experiment. On the chip a $6 \times 6$ matrix of $\alpha$-Lactalbumin spots is visible. Each group of three spots corresponds to a different polymer (c).

Figure 3: Anti- $\alpha$-Lactalbumin binding to two different modifications of $\alpha$-Lactalbumin spotted on a single IRIS chip. The solid lines in the inset (a) show a classic Langmuir fit of the data.

Figure 4: The curves in figure 3a, normalized by dividing every curve for the initial mass value.

Figure 5: Anti- $\alpha$-Lactalbumin binding to $\alpha$-Lactalbumin spotted on two flat-coated IRIS chips: one coated with Copoly Fluoride (light blue curve) and one with Copoly NAS 10\% Amphoteric (yellow). The solid lines in the inset (a) show a classic Langmuir fit of the data.

Figure 6 - Application of the localized functionalization method to the immobilization of LAC-1 peptide. (a) Anti- $\alpha$-Lactalbumin binding to two different modifications of LAC-1 spotted on a single IRIS chip. Each curve corresponds to a different spotted polymer. (b) The curves in Figure 6a, normalized by dividing each of them by the initial mass density value.

\section{List of tables}

Table 1: Mole percent of monomers used to synthesize polymers.

Table 2: ON and OFF rates obtained through a classic Langmuir fit of anti- $\alpha$-Lactalbumin binding on different $\alpha$-Lactalbumin modifications, spotted on top of different polymers spots.

Table 3: Initial mass density values for the $\alpha$-Lactalbumin spots on different polymers. 